\title{
Ultrastructural features of eosinophilic oesophagitis: impact of treatment on desmosomes
}

\author{
Kelley E Capocelli, ${ }^{1}$ Shahan D Fernando, $2,3,4,5$ Calies Menard-Katcher, ${ }^{2,3,4,5}$ \\ Glenn T Furuta, ${ }^{2,3,4,5}$ Joanne C Masterson, ${ }^{2,3,4,5}$ Eric P Wartchow $^{1}$
}

For numbered affiliations see end of article.

\section{Correspondence to Dr Kelley E Capocelli, Department of Pathology, Children's Hospital Colorado, 13123 East 16th Ave, Aurora, CO 80045, USA; Kelley. Capocelli@childrenscolorado. org}

KEC and SDF contributed equally.

JCM and EPW are joint senior authors.

Received 24 July 2014 Revised 4 October 2014 Accepted 9 October 2014 Published Online First 30 October 2014

\section{CrossMark}

$$
\begin{aligned}
& \text { To cite: Capocelli KE, } \\
& \text { Fernando SD, Menard- } \\
& \text { Katcher C, et al. J Clin } \\
& \text { Pathol 2015;68:51-56. }
\end{aligned}
$$

\section{ABSTRACT}

Aims A growing body of evidence suggests a role for altered epithelial barrier function in the pathophysiology of eosinophilic oesophagitis (EoE), but few have described the epithelial structure during inflammation. The purpose of this study was to define ultrastructural features of active, inactive EoE and control subject's oesophageal epithelia.

Methods We prospectively enrolled patients undergoing diagnostic upper endoscopy for evaluation of EoE. Mucosal pinch biopsies were obtained from the distal oesophagus and processed for routine histology and electron microscopic assessment. Clinical features of enrolled subjects were analysed and subjects were divided into four groups: normal, gastroesophageal reflux disease (GERD), inactive EoE and active EoE. Representative photomicrographs of the basal and superficial epithelia were reviewed for abnormalities. Desmosomes were quantified on the surface of epithelia three to four prickle-cell layers above the basal layer. Results Twenty-nine paediatric cases (ages 2-18 years) were enrolled in the study. We observed a significant decrease in the number of desmosomes per cell (DPC) of subjects with active EoE compared with inactive EoE, GERD and normal epithelia. With respect to DPC, no significant differences were found between inactive EoE compared with GERD or normal subjects. Additional ultrastructural features observed included epithelial microplicae and evidence of eosinophil transmigration, degranulation, and sombrero formation.

Conclusions Consistent with clinical and molecular findings, our ultrastructural data provide support for an altered oesophageal barrier in paediatric cases with active EoE, which may improve following treatment.

\section{INTRODUCTION}

Immunohistochemical and electron microscopic studies of oesophageal epithelium affected by eosinophilic oesophagitis (EoE) have focused on findings related to eosinophil activation and have identified a number of important characteristics including eosinophil degranulation, inverted granule protein cores and sombrero vesicle formations. ${ }^{1-4}$ While these findings document the impact of activated eosinophils as a functional endpoint, a growing body of evidence supports a role for altered barrier function in EoE. For example, expression of desmoglein, a cadherin protein associated with desmosome formation, is decreased in children affected by EoE. ${ }^{5}$ Filaggrin, a key barrier molecule, is dysregulated in the oesophageal epithelia of affected patients. ${ }^{6}$ Patterns of barrier dysfunction associated with active disease in adults with EoE have been observed using electrical impedance monitoring. ${ }^{7}$ Whether these alterations in the epithelia represent a primary causative feature or a reactive secondary event following eosinophilic inflammation is uncertain.

Based on these findings, we hypothesised that the oesophageal epithelia affected by EoE contained ultrastructural features of altered barrier function. Our data demonstrate that the number of desmosomes is decreased in the basal layer of stratified oesophageal epithelia of paediatric subjects with active EoE. Additionally, we present a number of other ultrastructural features present within the epithelia affected by EoE.

\section{METHODS}

We prospectively enrolled paediatric subjects between 1 year and 18 years of age undergoing upper endoscopy, to assess for EoE, from January 2011 to December 2012. Consecutive patients were approached to enrol in the study if they were undergoing initial endoscopic evaluation for $\mathrm{EoE}$ or repeat evaluation following treatment of $\mathrm{EoE}$ (topical steroids or diet). Clinical features of each subject were recorded and all underwent upper endoscopy with biopsy.

Subject groups were defined as follows:

- Active EoE was defined as subjects with symptoms of oesophageal dysfunction and $\geq 15$ eosinophils per high-power field (HPF) in whom alternative aetiologies of eosinophilia had been ruled out.

- Inactive EoE was defined as subjects with an established diagnosis of EoE who had undergone treatment with topical steroids or dietary exclusions, and whose symptoms resolved, and eosinophilia were found to be $<15$ eosinophils/HPF.

- Patients with gastroesophageal reflux disease (GERD) had symptoms related to reflux and $<15$ eosinophils/HPF.

- Normal persons had symptoms, such as abdominal pain, diarrhoea and vomiting significant enough to warrant an endoscopy, whose oesophageal histology was normal.

Tissue samples from the distal oesophagus were obtained and immediately fixed in cacodylatebuffered $2.5 \%$ glutaraldehyde and processed as previously described with samples being fixed in $2.0 \%$ osmium tetroxide then embedded in epoxy resin. ${ }^{8}$ Ultrathin sections were poststained with uranyl acetate and lead citrate and imaged using a Hitachi H-7650 TEM (Hitachi, Tokyo, Japan) equipped with an AMT XR-11 digital camera system (Advanced Microscopy Techniques, Massachusetts, USA). Samples were assessed without knowledge of their clinical diagnosis by two of the investigators (EPW and SDF). 
As described previously, we enumerated desmosomes within the epithelium by examining one to nine individual epithelial cells per patient that were three to four cell layers above the basal layer. $^{9-11}$ This region of interest was selected to maintain consistency in analysis. Desmosomes were identified and quantified as discrete, linear hyper-densities along the outer cellular membrane that make physical contact with an adjacent cellular membrane. Up to 43 cells were analysed within each of the four patient groups. Additional morphological features of the epithelia were identified independently by five investigators (EPW, KEC, JCM, SDF, GTF) who then reviewed their findings together.

Statistical significance was determined using a Kruskal-Wallis test and Dunn's test for multiple comparisons. Statistical analysis was performed using GraphPad Prism V.6.0d (GraphPad Software, La Jolla, California, USA).

\section{RESULTS}

Twenty-nine persons were enrolled in the study and clinical features are shown in table 1.

Typical endoscopic features of EoE, including linear furrows and exudates, as well as histological findings such as inflammation and basal cell hyperplasia, are seen in figure 1 . To minimise technical variability that may exist with assessing the stratified squamous epithelia in electron micrographs, we analysed the basal layer of the epithelia (figure $2 \mathrm{~A}$ ). The desmosome number was significantly reduced in epithelia with active EoE compared with normal epithelia $(7.6 \pm 3.7$ vs $14.1 \pm 5.9$, active EoE vs normal, $\mathrm{p}=0.0001$; figure $2 \mathrm{~B}$ ). Following standard EoE treatment, the desmosome number in inactive EoE (13.2 \pm 5.4$)$ was virtually the same as normal epithelia. Additionally, desmosome numbers in epithelia of patients with GERD (12.0 \pm 4.0$)$ were similar to inactive EoE and normal epithelia.

Further assessment of the intercellular space (ICS) revealed that the width of the ICS appears to be narrower in normal epithelia (figure 3A) compared with that observed in active EoE (figure $3 \mathrm{~B}$ ). The variability of the cell contours precludes the ability to fully assess this quantitatively. Desmosomes can be seen spanning the ICS in both normal and active EoE, but numbers are significantly reduced in active disease (figure 3C,

Table 1 Clinical features of patients

\begin{tabular}{|c|c|c|c|c|c|}
\hline & $\mathrm{N}^{*}$ & $\begin{array}{l}\text { Age } \\
\text { (years) }\end{array}$ & Symptoms & $\begin{array}{l}\text { Endoscopic } \\
\text { findingst }\end{array}$ & $\begin{array}{l}\text { Eos/ } \\
\text { HPF }\end{array}$ \\
\hline $\begin{array}{l}\text { Active } \\
\text { EoE }\end{array}$ & 9 & 2-15 & $\begin{array}{l}\text { Vomiting-5 } \\
\text { Abdominal } \\
\text { pain-4 }\end{array}$ & $\begin{array}{l}\text { F-6 } \\
\text { E-4 } \\
\text { LOVP-4 }\end{array}$ & $10-70$ \\
\hline $\begin{array}{l}\text { Inactive } \\
\text { EoE }\end{array}$ & 9 & $4-7$ & None & $\begin{array}{l}\text { F-1 } \\
\text { E-1 } \\
\text { LOVP-1 } \\
\text { N-6 }\end{array}$ & 0 \\
\hline GERD & 4 & $14-18$ & $\begin{array}{l}\text { Abdominal } \\
\text { pain-2 } \\
\text { Dysphagia-1 } \\
\text { Regurgitation-1 }\end{array}$ & $N-4$ & $0-3$ \\
\hline Normal & 7 & 9-17 & $\begin{array}{l}\text { Abdominal } \\
\text { pain-5 } \\
\text { Diarrhoea-1 } \\
\text { Vomiting-1 }\end{array}$ & $\begin{array}{l}\text { F-1 } \\
\text { LOVP-1 } \\
\text { FR-1 } \\
\text { N-5 }\end{array}$ & 0 \\
\hline
\end{tabular}

* Selected based on previous studies of epithelial features in EoE. ${ }^{12}$ tSome cases had more than one endoscopic finding.

E, exudate; EoE, eosinophilic oesophagitis; F, furrows; Fr, friable; HPF,

high-power-field; LOVP, loss of vascular pattern; N, normal.
D). When examining the superficial epithelia, several other observations were made. First, the ICS appears to be similar in width in normal and active EoE individuals even when a resting eosinophil is present (figure 4A, B). Second, microplicae or intercellular ridges were present on all epithelial surfaces (figure $4 \mathrm{~A}, \mathrm{~B})$ and appeared to form an intercellular 'zipper-like' pattern linking adjacent squamous epithelial cells.

Eosinophils, as described in previous reports, were observed resting (figure 5A), transmigrating (figure 5B) and degranulating (figure 5C, D). Figure 5B demonstrates a transmigrating eosinophil where the cytoplasmic membrane is shown to be intercalating between epithelial cells with varying degrees of contact. Activated eosinophils are shown to possess a number of key patterns, including extracellular extrusion of granules, secondary granules with inverted cores, sombrero vesicle formations and disruption of its cellular membrane (figure 5C, D).

\section{DISCUSSION}

Over the last decade, EoE has emerged as a leading cause of feeding dysfunction in young children and dysphagia and food impaction in teenagers and adults. ${ }^{13-15}$ Treatments, such as diet restrictions and topical steroids, are highly effective in inducing remission, and an increasing body of evidence identifies an underlying type- 2 cytokine-mediated pathogenetic pathway. Recent studies suggest that the epithelial barrier may play a critical role in the initiation and/or perpetuation of this inflammatory disease. As such, we performed a prospective study to determine ultrastructural features of the epithelial surface affected by EoE. This study reports a number of new observations related to ultrastructural epithelial features in children with EoE. Most prominent of these is that the number of desmosomes in subjects with active EoE is significantly decreased compared with inactive EoE, GERD and normal subjects.

Previous studies have identified ultrastructural epithelial abnormalities associated with peptic oesophagitis and oesophageal cancers, but ours is the first to identify epithelial ultrastructural findings in a prospective fashion in paediatric cases with active and inactive EoE. ${ }^{9}$ 16-21 Our findings bear particular relevance and support for recent studies that identify a role for epithelial barrier dysfunction in EoE. Through the use of electrical impedance, transepithelial resistance and fluorescent flux, van Rhijn et $\mathrm{al}^{7}$ reported diminished epithelial barriers in adults with EoE compared with those with normal epithelia. Katzka et $a l^{22}$ demonstrated increased dilated ICS associated with decreased expression of filaggrin, zonula occludens-1 and claudin-1 in oesophageal biopsies from patients with untreated EoE. Sherrill et $a l^{5}$ examined molecular regulation of barrier dysfunction and found that desmoglein-1 expression was decreased via a type- 2 cytokine microenvironment, and was associated with diminished barrier function in an ex vivo squamous epithelial model. In an electron micrograph, the number of desmosomes appears to be diminished, but enumeration was not presented. Here, we provide quantitative, morphological evidence that the oesophageal epithelial ultrastructure is indeed altered in EoE, a finding consistent with these functional and molecular studies. Additionally, we show that desmosome number returns to that found in the normal epithelium following treatment. This observation suggests that morphological evidence of barrier dysfunction occurs secondary to inflammation and not as a primary defect. Alternatively, treatments may repair underlying inherent epithelial barrier defects.

Our results also identify a number of interesting and important features related to relationships between the epithelium and eosinophils. First, while our findings identify the morphological 
Figure 1 Endoscopic and histological assessment of the oesophagus between normal patients and those with active eosinophilic oesophagitis (EoE), inactive EoE and GERD. $H \& E$ stained images obtained at $\times 10$ magnification. Scale bars represent $100 \mu$.

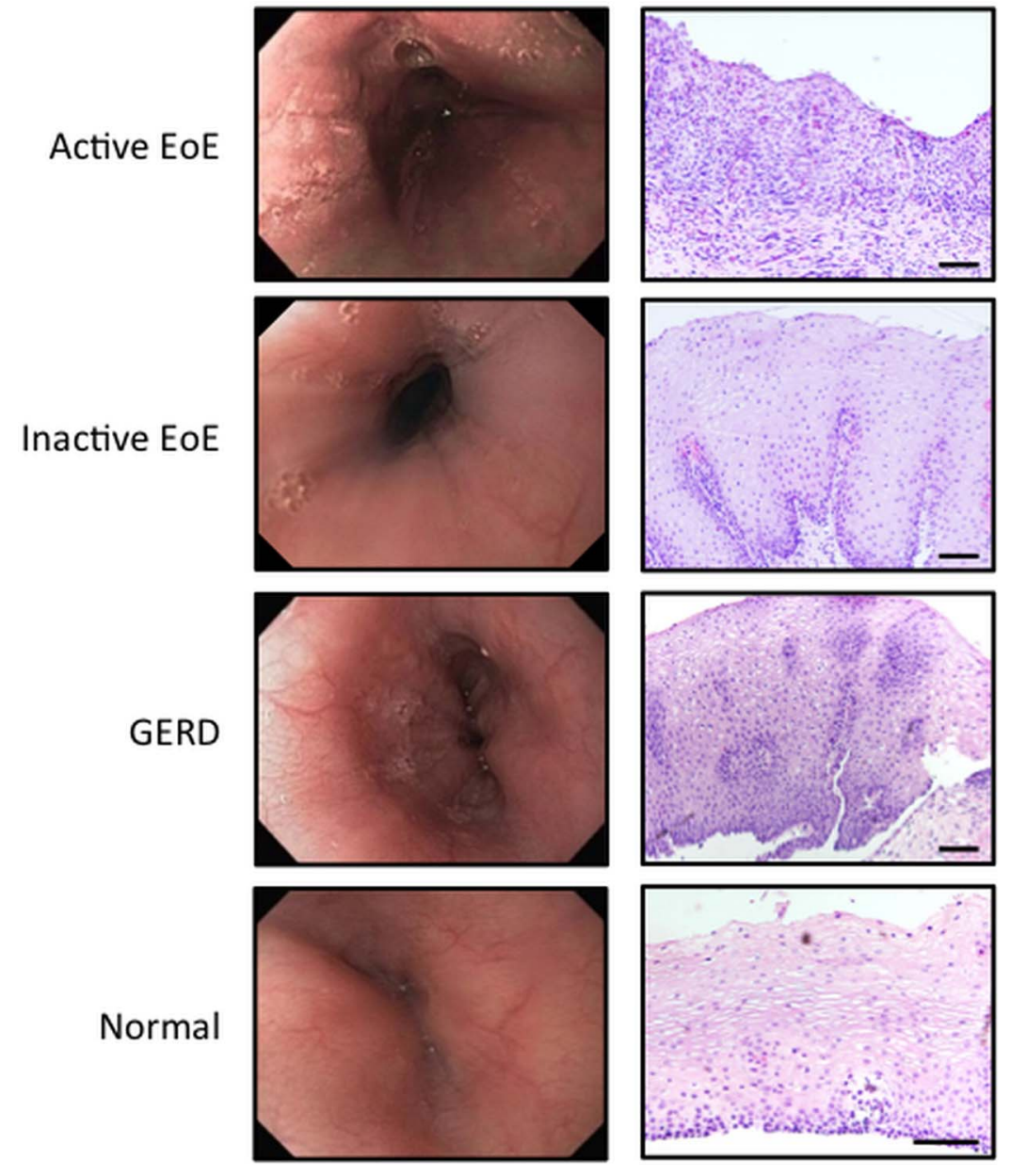

disruption of the normal stratified epithelia, our assessment also reveals the inherent nature of the oesophageal epithelium to maintain an intact barrier. We show that the superficial epithelia associated with active and inactive EoE, similar to that found in GERD and normal subjects, maintain a robust pattern of interdigitating microplicae or intercellular ridges. ${ }^{11}$ This 'zipper-like' morphology emphasises the importance of maintaining a barrier, which appears different than the basal layer, and further illustrates the pleotropic nature of the oesophageal epithelium.
Therefore, the superficial surface possesses an infrastructure comprised of flattened epithelial cells, microplicae, tight junctions and narrow ICS that creates a network to prevent luminal contents from penetrating the underlying immunomicromilieu. In EoE, the epithelia may limit exposure of the immune system to food allergens that initiate and perpetuate eosinophilic inflammation. Additionally, we provide evidence of the dynamic nature of eosinophil chemotaxis within the epithelia as seen in the figures demonstrating eosinophilic transmigration. Despite a
Figure 2 Desmosome quantification.

(A) Illustration of the oesophageal epithelium depicting the basal epithelial layer near the bottom and squamous epithelial layer near the top. The black box highlights the third prickle cell layer used to quantify desmosomes. (B) Number of desmosomes per cell in normal patients compared with those with GERD, active eosinophilic oesophagitis (EoE) and inactive EoE. Results are expressed as mean \pm SD.
A

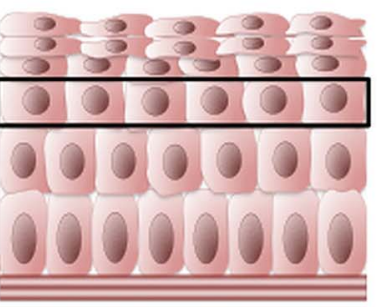

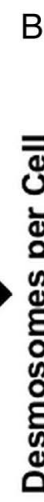

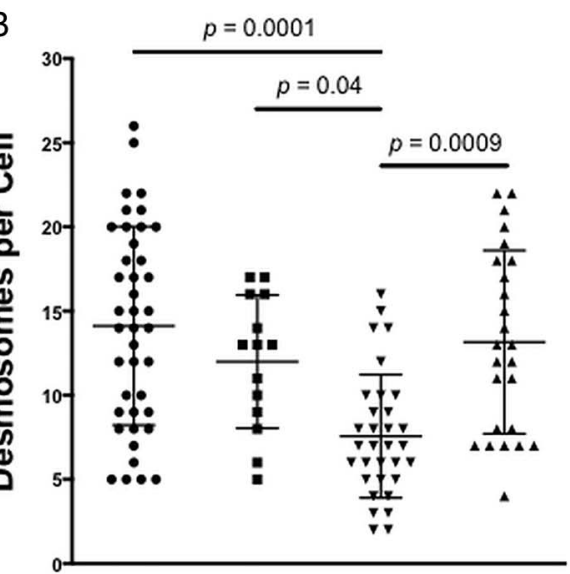

Normal GERD Active Inactive 

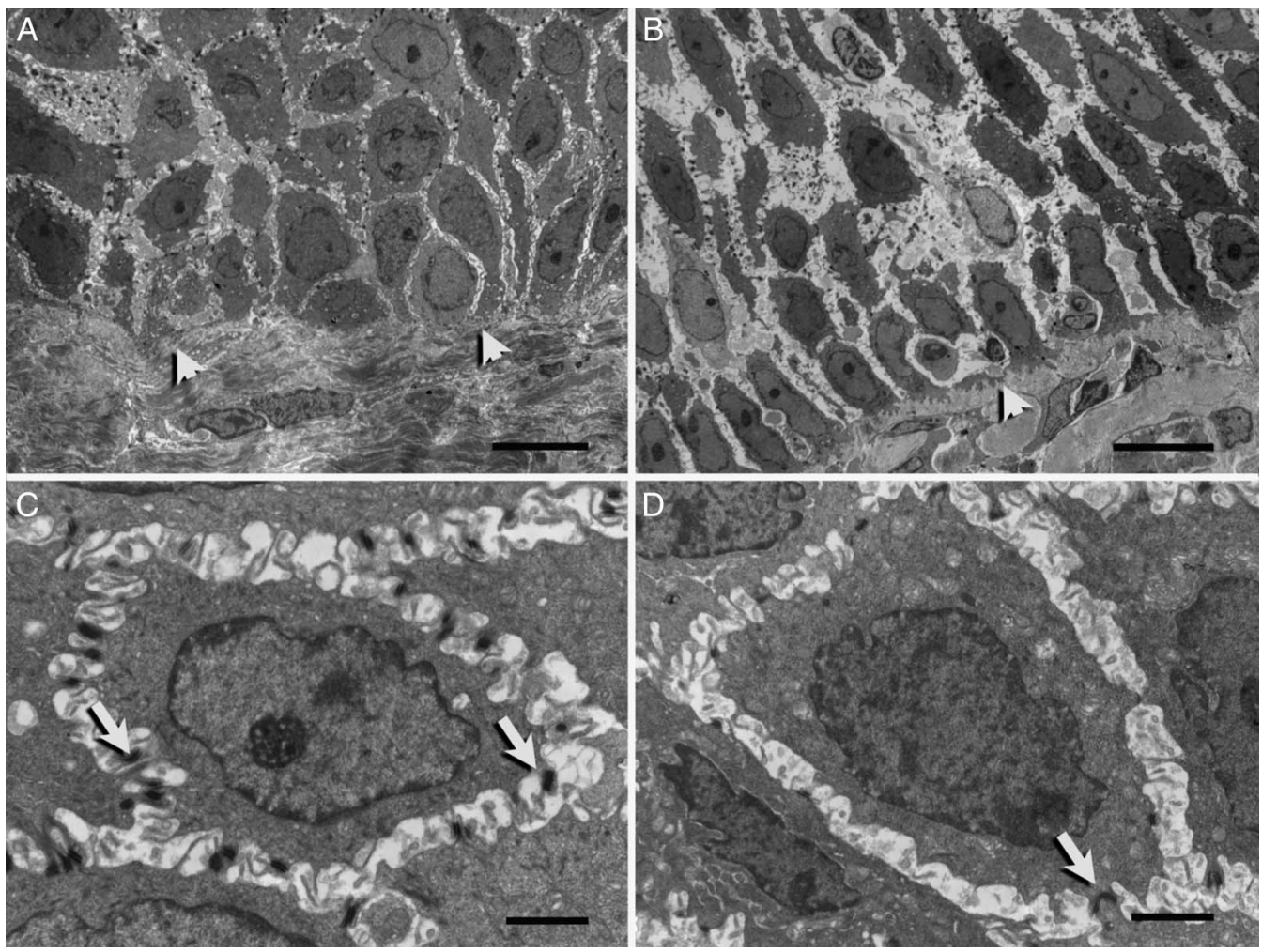

Figure 3 Electron micrographs of basal epithelial cells from normal patients $(A-C)$ and patients with active eosinophilic oesophagitis (B and D). $A$ and $B$ illustrate relative intercellular spaces. Arrowheads indicate the basal lamina separating basal cells from the lamina propria. (C and D) depict desmosomes (arrow) encircling basal cells located within the third prickle cell layer. Scale bars represent $10 \mu(A$ and $B)$ and $2 \mu$ (C and D).

number of studies defining the chemotactic pathways associated with EoE, none have captured an eosinophil actively migrating between the stratified squamous epithelia in EoE. ${ }^{23-25}$ Lastly, our ultrastructural analysis confirms recent observations that report the presence of eosinophil degranulation, sombrero vesicle formation and reversal of core proteins in EoE. ${ }^{1}$

While this ultrastructural report provides a high level of detail, it is difficult to provide a more expansive quantitative assessment or deeper mechanistic understanding than can be obtained through routine histological or molecular analysis, respectively. ${ }^{526} 27$ For instance, since eosinophils are unevenly distributed within the epithelia, quantification of their features and patterns of distribution in such a small field of view may not truly represent the overall mucosal inflammatory state. Therefore, we were limited to observational assessments only.
Similarly, we did not report exact measurements of the width of the ICS due to the high degree of variability in cell membrane shapes and forms.

Although enumeration of desmosomes within superficial squamous epithelia has been used in a number of previous studies to assess epithelial integrity and morphology, ${ }^{11} 192028$ our enumeration focused on the deeper, basal epithelial cells primarily due to technical concerns about the integrity of the superficial mucosa. Because of mechanical stresses on the epithelial surface caused by the pinch biopsy procedure, we could not consistently assess the uppermost superficial surfaces of the epithelia. However, we felt confident that the basal layer could be identified by allowing the underlying lamina propria to serve as a landmark. Thus, the numbers of desmosomes may appear to be sparse in the basal layer (figure 3) when compared with
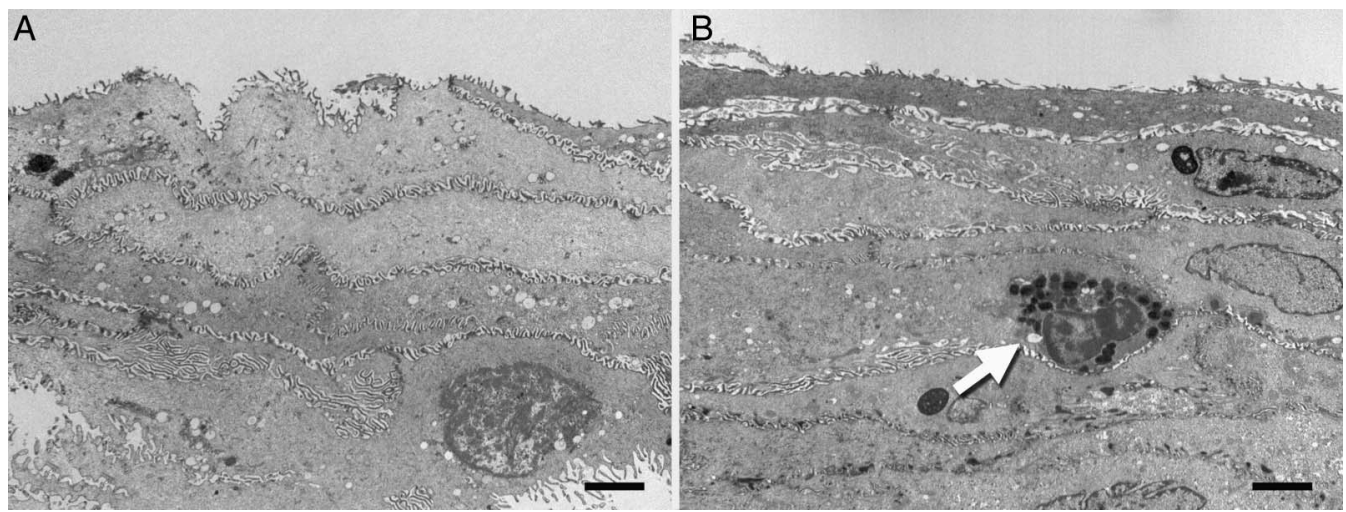

Figure 4 Electron micrographs of the stratified squamous epithelia in normal patients (A) and patients with active eosinophilic oesophagitis (B). A resting eosinophil (arrow) is depicted near the luminal surface. Scale bars represent $2 \mu$. 

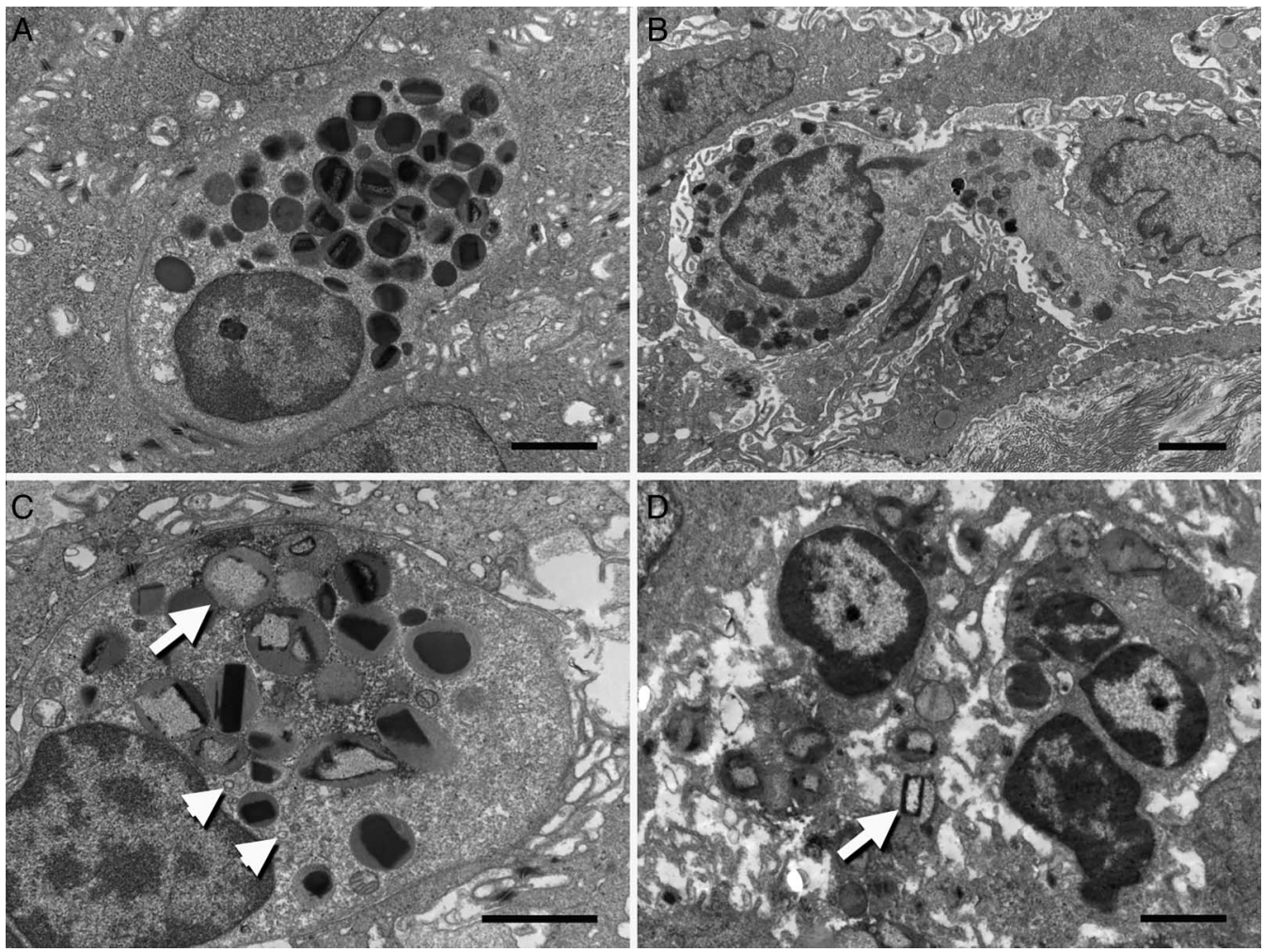

Figure 5 Electron micrographs of oesophageal epithelial eosinophils in various stages of activity including (A) resting state, (B) epithelial transmigration, (C) partial degranulation and (D) complete degranulation. Inverted granule protein cores (arrow) and sombrero formation (arrowhead) are seen within the degranulating eosinophils. Scale bars represent $4 \mu$.

those in other more superficial parts of the epithelia (figure 4). These findings may indicate that the more superficial epithelia require a larger number of desmosomes than the more basal epithelia to maintain barrier function for reasons discussed above, but this speculation would require further studies. Notwithstanding the technical challenges, we feel that the present study using transmission electron microscopy provides unique ultrastructural insights into features of EoE that would not be permitted through either routine histological or molecular techniques.

In summary, we report that the basal layer of stratified squamous epithelia appears to be altered in paediatric subjects with EoE. Future studies using laser capture microscopy to determine the exact molecular patterns leading to this finding will provide

\section{Take home messages}

- Oesophageal epithelial barrier dysfunction may play a critical role in the initiation and/or perpetuation of eosinophilic oesophagitis (EoE), a chronic immune-mediated inflammatory condition.

- At the ultrastructural level, a significant decrease in the number of desmosomes on the surface of the oesophageal epithelia of paediatric subjects with active EoE is observed. This provides support for recent works identifying evidence of altered barrier function in EoE.

- Eosinophils actively transmigrate through the oesophageal epithelium where they intimately adjoin with squamous epithelial cells prior to degranulation. more mechanistic insights and potential identification of novel therapeutic targets.

\section{Author affiliations}

${ }^{1}$ Department of Pathology, Children's Hospital Colorado, Aurora, Colorado, USA ${ }^{2}$ Section of Pediatric Gastroenterology, Hepatology and Nutrition, Digestive Health Institute, Children's Hospital Colorado, Aurora, Colorado, USA

${ }^{3}$ Gastrointestinal Eosinophilic Diseases Program, Department of Pediatrics, University of Colorado School of Medicine, Aurora, Colorado, USA

${ }^{4}$ Mucosal Inflammation Program, University of Colorado School of Medicine, Aurora, Colorado, USA

${ }^{5}$ University of Colorado School of Medicine, Aurora, Colorado, USA

Correction notice A sentence in Abstract section has been corrected since published Online First.

Acknowledgements The authors acknowledge and thank the physicians (Robert Kramer, Edward Hoffenberg, Edwin Liu, Edwin de Zoeten, Shikha Sundaram, Cara Mack, Michael Narkewicz, Ronald Sokol, Jason Soden, Deborah Neigut, David Brumbaugh and Christine Waasdorp Hurtado), nurses (Amy Kopp and Jo Anne Newton), research coordinators (Wendy Moore and Dresden Whitehead), technical staff (Bill Marcovich and Luann Goin), biostatistician (Zhaoxing Pan) and EM Laboratory Director (Gary Mierau) who contributed to this work. We are grateful to our patients and families who consented to be a part of this study.

Contributors Study concept and design (KEC, SDF, GTF, JCM, EPW); acquisition of data (KEC, SDF, CM-K, GTF, EPW); analysis and interpretation of data (KEC, SDF, GTF, $J C M, E P W)$; drafting of manuscript (SDF, GTF); critical revision of manuscript for important intellection content (KEC, SDF, CM-K, GTF, JCM, EPW); statistical analysis (SDF, JCM); obtained funding (GTF, JCM); study supervision (GTF, JCM, EPW).

Funding This study was supported by grants from the US National Institutes of Health K24-DK100303 (GTF) and T32-DK067009-09 (SDF), and American Partnership for Eosinophilic Disorders (JCM).

\section{Competing interests None.}

Ethics approval This study was approved by the Colorado Multi-Institutional Review Board.

Provenance and peer review Not commissioned; externally peer reviewed. 


\section{REFERENCES}

1 Saffari $H$, Hoffman LH, Peterson KA, et al. Electron microscopy elucidates eosinophil degranulation patterns in patients with eosinophilic esophagitis. J Allergy Clin Immunol 2014;6:1728-34.

2 Melo RCN, Dvorak AM, Weller PF. Electron tomography and immunonanogold electron microscopy for investigating intracellular trafficking and secretion in human eosinophils. J Cell Mol Med 2008;12:1416-19.

3 Friesen CA, Andre L, Garola R, et al. Activated duodenal mucosal eosinophils in children with dyspepsia: a pilot transmission electron microscopic study. J Pediatr Gastroenterol Nutr 2002;35:329-33.

4 Justinich CJ, Ricci AJ, Kalafus DA, et al. Activated eosinophils in esophagitis in children: a transmission electron microscopic study. J Pediatr Gastroenterol Nutr 1997;25:194-8.

5 Sherrill JD, KC K, Wu D, et al. Desmoglein-1 regulates esophageal epithelial barrier function and immune responses in eosinophilic esophagitis. Mucosal Immunol 2014;7:718-29.

6 Blanchard C, Stucke EM, Burwinkel K, et al. Coordinate interaction between IL-13 and epithelial differentiation cluster genes in eosinophilic esophagitis. J Immunol 2010;184:4033-41.

7 van Rhijn BD, Weijenborg PW, Verheij J, et al. Proton pump inhibitors partially restore mucosal integrity in patients with proton pump inhibitor-responsive esophageal eosinophilia but not eosinophilic esophagitis. Clin Gastroenterol Hepatol doi:10.1016/j.cgh.2014.02.037

8 Andrews PM. Microplicae: characteristic ridge-like folds of the plasmalemma. J Cell Biol 1976;68:420-9.

9 Liu C-C, Lee JW, Liu T-T, et al. Relevance of ultrastructural alterations of intercellular junction morphology in inflamed human esophagus.

J Neurogastroenterol Motil 2013;19:324-31.

10 Schluter $\mathrm{H}$, Moll I, Wolburg $\mathrm{H}$, et al. The different structures containing tight junction proteins in epidermal and other stratified epithelial cells, including squamous cell metaplasia. Eur J Cell Biol 2007;86:645-55.

11 Yassin Al TM, Toner PG. Fine structure of squamous epithelium and submucosal glands of human oesophagus. J Anat 1977;123:705-21.

12 Aceves SS, Newbury RO, Chen D, et al. Resolution of remodeling in eosinophilic esophagitis correlates with epithelial response to topical corticosteroids. Allergy 2010;65:109-16.

13 Straumann A, Aceves SS, Blanchard C, et al. Pediatric and adult eosinophilic esophagitis: similarities and differences. Allergy 2012;67:477-90.

14 Liacouras C, Furuta GT, Hirano I, et al. Eosinophilic esophagitis: Updated consensus recommendations for children and adults. J Allergy Clin Immunol 2011;128:3-20.
15 Furuta GT, Liacouras CA, Collins MH, et al. Eosinophilic esophagitis in children and adults: a systematic review and consensus recommendations for diagnosis and treatment. Gastroenterol 2007;133:1342-63.

16 Yan X, Li-ya Z, San-ren L. Dilated intercellular spaces in gastroesophageal reflux disease patients and the changes of intercellular spaces after omeprazole treatment. Chin Med J (Engl) 2008;121:1297-301.

17 Park S, Chun HJ, Jang JS, et al. Is Intercellular Space Different Among Layers in Normal Esophageal Mucosa? An Electron Microscopic Study. Dig Dis Sci 2011;56:3492-7.

18 Neumann H, Mönkemüller K, Fry LC, et al. Intercellular Space Volume Is Mainly Increased in the Basal Layer of Esophageal Squamous Epithelium in Patients with GERD. Dig Dis Sci 2010;56:1404-11.

19 Li F-Y. Interleukin-6, desmosome and tight junction protein expression levels in reflux esophagitis-affected mucosa. World J Gastroenterol 2009;15:3621-30.

20 Franchi A, Brogelli B, Massi D, et al. Dilation of intercellular spaces is associated with laryngo-pharyngeal reflux: an ultrastructural morphometric analysis of laryngeal epithelium. Eur Arch Otorhinolaryngol 2007;264:907-11.

21 Imamoto $\mathrm{H}$, Shiozaki $\mathrm{H}$, Tahara $\mathrm{H}$, et al. Disorder in ultrastructure of basement membrane and mechanical junction in human esophageal cancer. Med J Osaka Univ 1994;43:23-8.

22 Katzka DA, Tadi R, Smyrk TC, et al. Effects of topical steroids on tight junction proteins and spongiosis in esophageal epithelia of patients with eosinophilic esophagitis. Clin Gastroenterol Hepatol Published Online First: 27 Mar 2014. doi:10.1016/j.cgh.2014.02.039

23 Hogan SP, Mishra A, Brandt EB, et al. A critical role for eotaxin in experimental oral antigen-induced eosinophilic gastrointestinal allergy. Proc Natl Acad Sci USA 2000;97:6681-6.

24 Mishra A, Hogan SP, Brandt EB, et al. IL-5 Promotes eosinophil trafficking to the esophagus. J Immunol 2002;168:2464-9.

25 Blanchard C, Wang N, Stringer KF, et al. Eotaxin-3 and a uniquely conserved gene-expression profile in eosinophilic esophagitis. J Clin Invest 2006; 116:536-47.

26 Wex T, Mönkemüller K, Stahr A, et al. Gastro-oesophageal reflux disease is associated with up-regulation of desmosomal components in oesophageal mucosa. Histopathology 2012;60:405-15.

27 Villanacci V, Grigolato PG, Cestari R, et al. Dilated intercellular spaces as markers of reflux disease: histology, semiquantitative score and morphometry upon light microscopy. Digestion 2001;64:1-8.

28 Logan KR, Hopwood D, Milne G. Cellular junctions in human oesophageal epithelium. J Pathol 1978;126:157-63. 